\title{
Antifungal activity of paracloacal gland secretion of Caiman yacare (DAUDIN, 1802)
}

The objective of this study was to evaluate the in vitro antifungal activity of ethanolic and aqueous extracts obtained from Caiman yacare paracloacal gland (PG) against Candida albicans (ATCC 10231), as well as a bibliographical survey on the chemical composition of exudates of PG. The PG were collected from the disposal generated during the slaughter of C. yacare by regularized industry. Two extracts were made from these glands, one ethanolic and the other aqueous. The Minimal Inhibitory Concentration (MIC) of the substances were determined by dilution of the extract in series using the microdilution technique in the culture medium Sabouraud broth, carried out in a 96-well microplate visually read after 48 hours of incubation, confirmed by the method using $0.01 \%$ aqueous resazurin dye. The ethanolic extract had MIC at the concentration of $25 \mu \mathrm{g} / \mathrm{L}$. The Minimum Fungicidal Concentration (MFC) was determined confirmed by the method using $0.01 \%$ aqueous resazurin dye. The ethanolic extract had MIC at the concentration of $25 \mu \mathrm{g} / \mathrm{L}$. The Minimum Fungicidal Concentration (MFC) was determined
by subcultures of MIC in Sabouraud agar medium. The ethanolic extract presented MFC at a concentration of $50 \mu \mathrm{g} \mathrm{/} \mathrm{mL.} \mathrm{The} \mathrm{aqueous} \mathrm{extract} \mathrm{showed} \mathrm{no} \mathrm{antifungal} \mathrm{activity} \mathrm{at} \mathrm{the}$ concentrations tested. This work is the first work to assess an activity of the PG secretion and reveals pharmacological potential in a local product previously discarded.

\section{Atividade antifúngica da secreção de glândulas paracloacais de Caiman yacare (DAUDIN, 1802)}

Este estudo teve como objetivo avaliar a atividade antifúngica in vitro dos extratos etanólico e aquoso do exsudado obtido a partir glândula paracloacal (PG) de Caiman yacare frente a Candida albicans (ATCC 10231), bem como foi realizado um levantamento bibliográfico sobre a composição química de exsudatos de PG. As PG foram coletadas do descarte gerado durante o abate de C. yacare por frigorífico regularizado. Dessas glândulas foram confeccionados dois extratos, um etanólico e outro aquoso. A Concentração Inibitória Mínima (MIC) das substâncias, foram determinadas por meio da diluição do extrato em série através da técnica de microdiluição no meio de cultura caldo Sabouraud, realizado em placa de 96 poços com leitura verificada após 48 horas de incubação, confirmada pelo método visual utilizando corante de solução aquosa de resazurina a 0,01\%. 0 extrato etanólico apresentou MIC na concentração de $25 \mu \mathrm{g} / \mathrm{mL}$. A Concentração Fungicida Mínima (MFC) foi determinada por subculturas da MIC em meio de cultura ágar Sabouraud. O extrato etanólico apresentou MFC na concentração de 50 $\mu \mathrm{g} / \mathrm{mL}$ $\mathrm{O}$ extrato aquoso não apresentou atividade antifúngica nas concentrações testadas. Esse trabalho é o primeiro a aferir uma atividade da secreção da PG e revela potencial farmacológico em um produto local até então descartado.

Keywords: Pantanal; Jacaré; Candida albicans; Crocodilianos; Leveduras.

Topic: Uso de Recursos Naturais

Reviewed anonymously in the process of blind peer.
Received: 02/02/2018

Approved: 20/05/2018
Lucas Polizzeli Azevedo (iD

Universidade do Estado do Mato Grosso, Brasil http://lattes.cnpq.br/4839407879193826 http://orcid.org/0000-0002-5704-5950

lucasjauru@gmail.com

Leandro Nogueira Pressinotti (ib)

Universidade do Estado do Mato Grosso, Brasil http://lattes.cnpq.br/7446524133090464 http://orcid.org/0000-0003-2682-1598

microtomo@unemat.br

\section{Rhavena Graziela Liotti (iD}

Instituto Federal de Mato Grosso, Brasil http://lattes.cnpq.br/8935685390633972 http://orcid.org/0000-0001-8492-8823 rhavena.liotti@cas.ifmt.edu.br

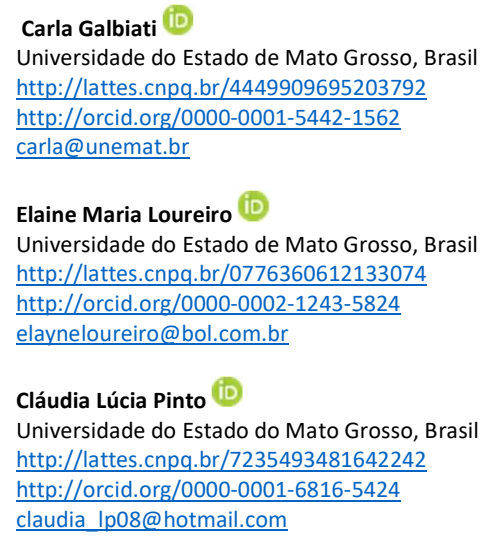

Natasha Rayane de Oliveira Lima Universidade do Estado do Mato Grosso, Brasil http://lattes.cnpq.br/9449797453342327 http://orcid.org/0000-0002-4547-0263 natasharayane@hotmail.com

Mariana Lenina Menezes Aleixo (iD Universidade do Estado do Mato Grosso, Brasil http://lattes.cnpq.br/2641517259797926 http://orcid.org/0000-0002-9363-2423 marianaaleixo@uol.com.br

\section{Referencing this:}

AZEVEDO, L. P.; PRESSINOTTI, L. N.; LIOTTI, R. G.; GALBIATI, C.; LOUREIRO, E. M.; PINTO, C. L.; LIMA, N. R. O.; ALEIXO, M. L. M. Atividade antifúngica da secreção de glândulas paracloacais de Caiman yacare (DAUDIN, 1802). Revista Ibero-Americana de Ciências Ambientais, v.9, n.1, p.140-151, 2018. DOI: http://doi.org/10.6008/CBPC2179-6858.2018.001.0010 


\section{INTRODUCTION}

The launch of new antibiotics decreased in last years, and reports of resistance to antibiotic therapy increased (GUIMARÃES et al., 2010). Among pathogens, there are microorganisms able to resist the human defense mechanisms, in addition, the incorrect use of chemotherapeutic favors the emergence of resistant microorganisms (GIAMARELLOU, 2006; TORTORA et al., 2012).

Bioprospection of essential oils from plants and zoological products is growing worldwide (BAYDAR et al., 2004; INOUYE et al., 2006), therefore, due to high biodiversity, Brazil can occupy a protagonist role in the scenario (KATE et al., 1999). A potential starting point for bioprospection can be the traditional communities, that even before the advent of antibiotic therapy, treated their patients through the use of local flora and fauna (ALBERNAZ et al., 2010; BARROS et al., 2012; MENALE et al. 2016; OLIVEIRA et al., 2010).

By-products obtained from parts of crocodilians, are frequently described by traditional knowledge, being the species Paleosuchus palpebrosus and Caiman crocodiles with greater presence (ALVES et al., 2010). The use of fresh fats of Caiman latirostris, Melanosuchus niger and P. palpebrosus for treatments of rheumatism (ALVES et al., 2008); use of fresh fat from C. crocodilus for treatment of pneumonia (BARROS et al., 2012); the use of fat of $C$. latirostris as healing (SOUTO et al., 2011) or for treatment of asthma (OLIVEIRA et al., 2010).

There are some scientific results regarding the use of by-products obtained from crocodilians, such as the anti-inflammatory and antimicrobial action of Crocodylus niloticus oil. (BUTHELEZI et al., 2012), antibacterial activity of the serum protein profile of Alligator mississippiensis (MERCHANT et al., 2003) Amoebicidal effect of $A$. mississippiensis serum (MERCHANT et al., 2004), a high antimicrobial activity of the leukocyte extract of $A$. mississipiensis (MERCHANT et al., 2006), antimicrobial and anti-inflammatory activities in C. niloticus oil (BUTHELEZI et al., 2012), antimicrobial and anti-inflammatory activity of the blood extracts of Crocodylus siamensis (PATA et al., 2011; KOMMANEE et al., 2012; KOMMANEE et al., 2014).

In the Pantanal, there is the cultivation of Caiman yacare (Jacare do Pantanal), which represents a diversification of zootechnical and economic activities in the region. The main products commercialized are leather and meat, however, there are efforts to valorize by-products, such as the production of hamburgers, sausages and mortadella using less noble meats (FERNANDES et al., 2014; MORAIS et al., 2013; ROMANELLI et al., 2002).

The pharmacological potential of crocodilian by-products can be a promising area, adding economic value to the $C$. yacare productive chain. During the slaughter there is generation of discard of viscera, and between them there is Paracloacal Gland (PG). The exudate of PG from the crocodilians has many molecules on their chemical composition, and some of them can exert the antifungal effect in cloaca.

Finally, yeasts of Candida albicans is the cause of most fungal infections, which is considered an opportunistic infection that affects predisposed individuals, usually those with inefficient immune systems or who have been subjected to prolonged antibiotic treatment (LIMA et al., 2006; ZHANG et al., 2002). 
In this work, it was evaluated the fungicidal activity of $C$. yacare PG exudate against Candida albicans (ATCC 10231), as well as a bibliographic survey on the chemical composition of PG exudate from many species of crocodilians.

\section{MATERIAL AND METHODS}

The research was carried out in partnership with the company regularly authorized by the current standard, which carries out the slaughter of $C$. yacare (SISBio 58681-1). We collected 30 PG from C. yacare measuring over 1.30 meters in length. The PG were submitted to mechanical asepsis with washing in running water and chemical asepsis with three times of 5 minutes baths in $70 \%$ alcohol, a bath with $2.0 \%$ sodium hypochlorite for 5 minutes and three baths with sterile distilled water for 5 minutes.

Glandular secretion was extracted by manual compression of the PG capsule in a mortar (WELDON et al., 1989; WILLIAMS et al., 1989). The exudate was drained and preserved in a sterile refrigerated bottle. For the antifungal test, two extracts were prepared, one ethanolic and one aqueous.

The exudates were added in two flasks containing, respectively, ethanol (99.8\%) and sterile distilled water, in the proportion of $50 \%$ exudate and $50 \%$ diluent. The flasks were shaken daily and, after 14 days, the two solutions were filtered on filter paper and $1 \mathrm{~mL}$ of the filtrate was dried in vacuo. The sample was considered free of diluent when the weight did not decrease among successive weighing.

The extracts of PG were respectively resuspended in ethanol and distilled water at the final concentration of $800 \mu \mathrm{g} / \mathrm{mL}$ (ALVARENGA et al., 2007; BAYDAR et al., 2004; NASCIMENTO et al., 2007; OLIVEIRA et al., 2011; SANTOS et al., 2010).

For the evaluation of the antifungal activity, a lineage of Candida albicans (ATCC 10231) was used, which was suspended in $0.85 \%$ saline solution, comparing with the MacFarland 0.5 scale, as a standardization of inoculum density, which represents $1,5 \times 10^{6}$ Colony Forming Units (UFC / mL) (CLSI, 2012).

The antifungal activity in the Minimum Inhibitory Concentration (MIC) of the elaborated extracts was determined by the broth microdilution method (CLSI, 2008; 2012) adapted. Aliquots of $100 \mu \mathrm{L}$ of the PG extract suspension were added to $100 \mu \mathrm{L}$ of Sabouraud broth culture medium in the first well of the 96 well plate "lines" and the serial (1: 2) dilution was performed, resulting in concentrations of 400, 200, 100, 50, 25, $12.5,6.25,3.12,1.56$ and $0.78 \mu \mathrm{g} / \mathrm{mL}$. Subsequently, $10 \mu \mathrm{L}$ of standardized inoculum $1.5 \times 10^{6}$ (UFC / mL) was added to each well (CLSI, 2008; 2012).

The antibiotic Itraconazole $100 \mu \mathrm{g} / \mathrm{mL}$ was used as antifungal control. The control of diluent effect was carried out by ethanol $2 \%(\mathrm{v} / \mathrm{v})$ assay. Sterility control of the medium was carried out by observing the culture medium Sabouraud broth without any interference.

The microplates were incubated for 48 hours at $37^{\circ} \mathrm{C}$, after the incubation period $20 \mu \mathrm{L}$ of $0.01 \%$ aqueous resazurin solution was added to each well and reincubated for 6 hours at $37^{\circ} \mathrm{C}$, after the incubation time with resazurin the microplates were analyzed.

Resazurin was used because the aqueous and ethanolic extract of PG acquired a cloudy coloration that makes difficult the optical reading, therefore the chromatic conversion of resazurin is the most suitable 
for oils extracted from PG. Classically, the resazurin indicates absence of microorganism growth when keep the blue color into the well at the end of the protocol (ALVES et al., 2008) and the MIC was defined as the lowest concentration of the tested solution that prevented the growth of the microorganism (NATECHE, 2006; PALOMINO et al., 2002).

After visual reading of the microplate, $10 \mu \mathrm{L}$ were removed from the last three concentrations of microplates wells that keep the blue resazurin color, and seeded in Petri dishes containing Sabouraud agar, and then the plates were incubated for an additional 48 hours at $37^{\circ} \mathrm{C}$ for determination of Minimum Fungicidal Concentration (MFC). The concentrations with growth in the Petri dish was considered as fungistatic action (ELLOF, 1998), and the concentration with absence of growth was considered MFC.

The antimicrobial activity was classified as good activity when MIC $<100 \mu \mathrm{g} / \mathrm{mL}$ and MIC moderated between 100 and $500 \mu \mathrm{g} / \mathrm{mL}$ (HOLETZ et al., 2002). The assays were performed in triplicate.

\section{RESULTS}

The PG is located in the distal part of the digestive system, inserted in the wall of the cloaca, exhibit an oval shape, pink color, with an aperture to cloaca lumen. The largest glands belonging to males, surrounded by capsular musculature. The exudate has a golden yellow color, is viscous, with a strong odor that disperses easily in the environment.

The yield of the ethanolic extract was $43.00 \%$ and the yield of the aqueous extract was $83.03 \%$ of the volume of the dried filtrate under vacuum.

The aqueous extract exhibit resazurin conversion from blue to pink on all tested concentrations of 400 to $0.78 \mu \mathrm{g} / \mathrm{mL}$. The ethanolic extract exhibit no resazurin conversion on 400 to $25 \mu \mathrm{g} / \mathrm{mL}$, considering MIC of $25 \mu \mathrm{g} / \mathrm{mL}$ for $C$. albicans. Wells with concentrations below $25 \mu \mathrm{g} / \mathrm{mL}$ exhibit resazurin conversion. Ethanolic and sterile control exhibit no resazurin conversion.

The agar incubation of ethanolic extract assay exhibit death of $99,9 \%$ of $C$. albicans at MFC of $50 \mu \mathrm{g}$ / $\mathrm{mL}$, and the concentration of $25 \mu \mathrm{g} / \mathrm{mL}$ was considered fungistatic once it presents a few growth of $C$. albicans (Table 1). In ethanol control the inoculum remained viable throughout the experiment period.

Table 1: MIC and MFC ( $\mu \mathrm{g} / \mathrm{ml})$ of extracts of PG of C. yacare.

\begin{tabular}{cccc}
\hline & \multicolumn{3}{c}{ Microorganism } \\
\cline { 2 - 4 } Extracts & MIC & Candida albicans \\
\cline { 2 - 4 } & NC & NC \\
\hline AEPGO & 25 & 50 \\
PGOEE & & \\
\hline
\end{tabular}

AEPGO - Aqueous Extract of Paracloacal Gland Oil; PGOEE - Paracloacal Gland Oil Ethanol Extract; NC - Did not get results in the tested Concentrations.

\section{DISCUSSION}

The knowledge of traditional communities in the search for bioactive molecules may represent a promising starting point for pharmaceutical research (PINTO et al., 2002; SANT'ANA, 2002), so that about 
$50 \%$ of current medicines were developed from natural resources. In this sense, there is a growing interest in the pharmaceutical industry for bioprospecting these natural resources (JUNIOR, 2010).

Adding value to caiman's products and by-products helps make zootechnical activity even more feasible, a form of incentive to maintain a renewable resource rather than exploit and destroy existing populations, promoting population interests to produce and preserve the biome in which the species is inserted (BLAKE et al., 1975; MOURÃO, 2000).

This article characterizes an antifungal activity evidenced by in vitro tests against the yeasts $C$. albicans. The results are consistent with those found in crocodile by-products, such as in $C$. niloticus oil, with ample antibacterial activity and marked fungicidal activity against C. albicans (BUTHELEZI et al., 2012); Antibacterial and antifungal activity against $C$. albicans by leukocytes extracts of $C$. siamenses (PATA et al., 2011) and a wide antimicrobial activity of leucocyte extract from A. mississippiensis (MERCHAN et al., 2006).

The good antimicrobial activity was obtained from a crude oil with low solubility in culture medium. In order to improve the quality of the experiments with essential oils, solvents, detergents, or emulsifying agents, such as ethanol, are used to facilitate their solubility in the culture medium (NASCIMENTO et al., 2007). In this study the ethanolic control did not present inhibitory activity against the inoculum result similar to other studies (ALVARENGA et al., 2007; BAYDAR et al., 2004; NASCIMENTO et al., 2007; OLIVEIRA et al., 2011; SANTOS et al., 2010).

The absence of antifungal activity of aqueous extract, in comparison to ethanolic extract, can be explained by the polarity of the solvent, once water and ethanol solubilizes different molecules according to hydrophobicity affinity.

Based on the bibliographical references consulted about the chemical composition of the paracloacal secretion, we found 234 different substances, described by 8 articles that deal with chemical compositions in several species of crocodilians. In A. mississippiensis were described 27 molecules, Alligator sinensis 55 molecules, C. crocodilus 60 molecules, C. latirostris 41 molecules, C. yacare 23 molecules, Crocodylus acutus 68 molecules, P. palpebrosus 43 molecules and Paleosuchus trigonatus 36 molecules (DUNN et al., 1993; GARCÍA-RUBIO et al., 2002; KRUCKERT et al., 2006; MCDANIEL et al., 1998; WELDON et al., 1989; WELDON et al., 1988, 1989; WHEELER et al., 1999). Among the molecules there were esters, alcohol, carboxylic acids, ketones, aldehydes and hydrocarbons. The esters were the most frequently described molecule, followed by ketone and alcohol. C. yacare presented the lowest number of molecules described (Table 2). Later works will describe other substances present in the ethanolic extract and pointed out the substance responsible for the antifungal activity.

Table 2: Molecules found at paracloacal glands of Alligator mississippiensis, Alligator sinensis, Caiman crocodilus, Caiman latirostris, Caiman yacare, Crocodylus acutus, Paleosuchus palpebrosus and Paleosuchus trigonatus.

\begin{tabular}{|c|c|c|c|c|c|c|c|c|}
\hline ÉSTERES & $\begin{array}{c}\text { A. } \\
\text { sinensis }\end{array}$ & $\begin{array}{c}\text { A. } \\
\text { mississippiensis }\end{array}$ & C. crocodilus & $\begin{array}{c}\text { C. } \\
\text { latirostris }\end{array}$ & $\begin{array}{c}\text { C. } \\
\text { yacare }\end{array}$ & $\begin{array}{c}P . \\
\text { palpebrosus }\end{array}$ & P. trigonatus & $\begin{array}{c}\text { C. } \\
\text { acutus }\end{array}$ \\
\hline 10-Heptadecenyl acetate & + & & & & & & & \\
\hline 11-Octadecenyl acetate & + & & & & & & & \\
\hline $\begin{array}{l}\text { 3,7-Dimethyl-6-octen-1-yl } \\
\text { (acetato de citronelilo) }\end{array}$ & & & + & & & + & & \\
\hline 3-Dodecenyl acetate & + & & & & & & & \\
\hline
\end{tabular}


4,8-Heptadecadieny

acetate

4-Tridecenyl acetate

5,9-Octadecadienyl

acetate

5-Hexadecenyl acetate

7-Hexadecenyl formate

8-Heptadecenyl acetate

8-Pentadecenyl acetate

9-Octadecenyl acetate

Acetate dihidrofarnesil

Butanoato de citronelilo

Cholesteryl formate

Citronellyl acetate

Citronellyl butyrate

Citronellyl format

Citronellyl butanoate

Citronellyl pentanoate

Decyl 3-methylbutanoate

Decyl acetate

Decyl tetradecanoate***

DHF 3-methylbutanoate

DHF acetate

DHF butenoate

DHF dodecanoate

DHF heptadecenoate

DHF hexadecenoate

DHF hexanoate

DHF octadecanoate

DHF octadecenoate

DHF octanoate

DHF pentadecenoate

DHF propanoate

DHF tetradecanoate

DHF tetradecenoate

DHF tridecanoate

DHF undecanoate

Dodecyl 3-

methylbutanoate

Dodecyl acetate

Dodecyl butanoate

Dodecyl dodecanoate

Dodecyl formate

Dodecyl hexadecanoate

Dodecyl hexanoate

Dodecyl octadecanoate

Dodecyl oleate

Dodecyl pentanoate

Dodecyl tetradecanoate

Eicosenyl acetate

Eicosenyl formate

Eicosyl acetate

Heptadecenyl butyrate

Heptadecadienyl acetate

Heptadecadienyl formate

Heptadecenyl acetate

Heptadecenyl acetate

Heptadecenyl butanoate

Heptadecenyl butyrate

Heptadecenyl formate

Heptadecyl acetate

Heptadecyl butanoate

Heptadecyl butyrate

Heptadecyl formate

Hexadecadienyl acetate

Hexadecenyl acetate

Hexadecenyl butanoate

+
+
+
+
+
+
+
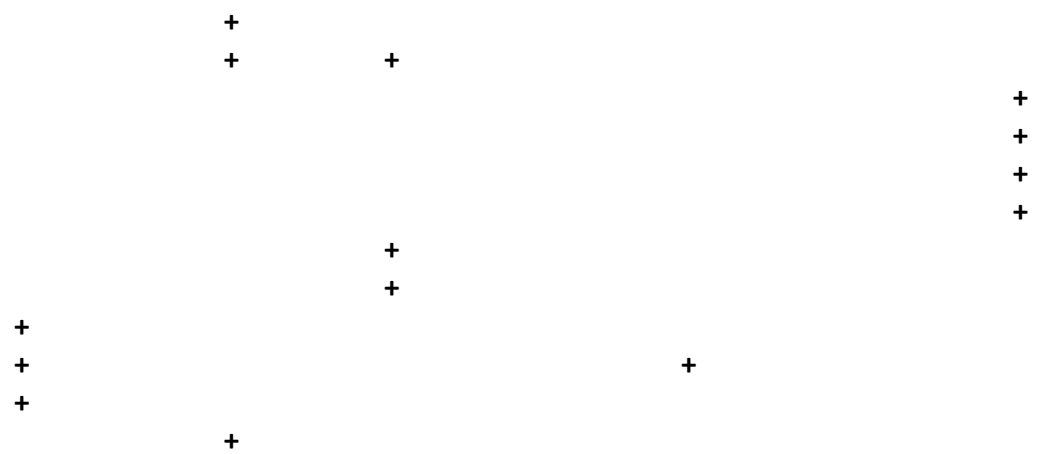

$+$

$+$

$+$

$+$

$+$

$+$

$+$

$+$

$+$

$+$ 
Hexadecenyl butyrate

Hexadecenyl propanoate

Hexadecyl acetate

Hexadecyl butanoate

Hexadecyl caproate

Hexadecyl caprylate

Hexadecyl formate

Hexadecyl pentanoate

Hexadecyl propanoate

Hexadecyl propanoate

Citronellyl hexanoate

Methyl palmitate

Nonadecyl acetate

Nonyl acetate

Octadecadienyl acetate

Octadecadienyl butyrate

Octadecenyl acetate

Octadecenyl butanoate

Octadecenyl butyrate

Octadecenyl formate

Octadecyl acetate

Octadecyl butanoate

Octadecyl butyrate

Octadecyl formate

Octyl Oleate

Pentadecadienyl acetate

Pentadecane

Pentadecenyl acetate

Pentadecenyl butanoate

Pentadecenyl butyrate

Pentadecenyl formate

Pentadecyl acetate

Pentadecyl butanoate

Pentadecyl butyrate

Pentadecyl caproate

Pentadecyl formate

Pentadecyl pentanoate

Tetradecenyl acetate

Tetradecenyl butanoate

Tetradecenyl butyrate

Tetradecyl 3-

methylbutanoate

Tetradecyl acetate

Tetradecyl butanoate

Tetradecyl butyrate

Tetradecyl dodecanoate

Tetradecyl formate

Tetradecyl hexadecanoate

Tetradecyl pentanoate

Tetradecyl propanoate

Tetradecyl

tetradecanoate

Tridecyl acetate

Tridecyl butanoate

Tridecyl butyrate

$x, y$-Heptadecadienyl

acetate

$x, y$-Hexadecadienyl

acetate

$x, y$-Octadecadienyl

acetate

$\mathrm{x}, \mathrm{y}$-Tetradecadienyl

acetate

x-Pentadecenyl acetate

z,w-Heptadecadienyl

acetate

$+$

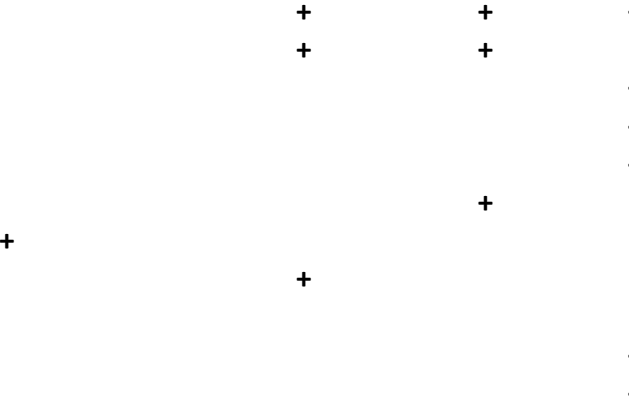

$+$

$+$
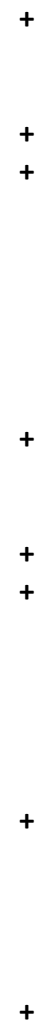
z,w-Pentadecadienyl acetate

\begin{tabular}{|c|c|c|c|c|c|c|c|c|}
\hline ETANOL & $\begin{array}{c}\text { A. } \\
\text { sinensis }\end{array}$ & $\begin{array}{c}\text { A. } \\
\text { mississippiensis }\end{array}$ & $\begin{array}{c}\text { C. } \\
\text { crocodilus }\end{array}$ & $\begin{array}{c}\text { C. } \\
\text { latirostris }\end{array}$ & $\begin{array}{c}\text { C. } \\
\text { yacare }\end{array}$ & $\begin{array}{c}P . \\
\text { palpebrosus }\end{array}$ & $\begin{array}{c}\text { P. } \\
\text { trigonatus }\end{array}$ & $\begin{array}{c}\text { C. } \\
\text { acutus }\end{array}$ \\
\hline 10-Heptadecen-1-ol & + & & & & & & & \\
\hline 11-Octadecen-1-ol & + & & & & & & & \\
\hline 2,3-Dihidrofarnesol & & & + & & & & & \\
\hline $\begin{array}{l}\text { 3,7-Dimetil-6-octen-1-ol } \\
\text { (citronelol) }\end{array}$ & & & & + & & + & + & \\
\hline 8-Heptadecen-1-ol & + & & & & & & & \\
\hline Decanol & & & & & & + & + & \\
\hline 3-Dodecen-1-ol & + & & & & & & & \\
\hline Dodecanol & + & & & & & & + & \\
\hline $\begin{array}{l}\text { u,v-Heptadecadiendien-1- } \\
\text { ol }\end{array}$ & + & & & & & & & \\
\hline Heptadecadienyl alcohol & & & & & & & & + \\
\hline Heptadecanol & + & & & & & & & \\
\hline Heptadecenol & & & & & & & + & \\
\hline $\mathrm{x}, \mathrm{y}$-Heptadecdien-1-ol & + & & & & & & & \\
\hline Heptadecyl alcohol & & & & & & & & + \\
\hline z,w-Heptadecadien-1-ol & + & & & & & & & \\
\hline Hexadec-5-an-1-ol & + & & & & & & & \\
\hline Hexadec-9-an-1-ol & + & & & & & & & \\
\hline Hexadecanol & + & & & & & & + & \\
\hline Hexadecanol & & & & & & + & & \\
\hline Hexadecenyl alcohol & & & & & & & & + \\
\hline x,y-Hexadecadien-1-ol & + & & & & & & & \\
\hline Hexadecyl alcohol & & & & & & & & + \\
\hline Nonanol & & & & & & + & & \\
\hline Nonenol & & & & & & & + & \\
\hline 5,9-Octadecadien-1-ol & + & & & & & & & \\
\hline 9-Octadecen-1-ol & + & & & & & & & \\
\hline Octadecanol & + & & & & & + & & \\
\hline Octadecenol & & & & & & & + & \\
\hline Octadecenyl alcohol & & & & & & & & + \\
\hline x,y-Octadecadien-1-ol & + & & & & & & & \\
\hline Octadecyl alcohol & & & & & & & & + \\
\hline Octanol & & & & & & & + & \\
\hline 8-Pentadecen-1-ol & + & & & & & & & \\
\hline Pentadecanol & & & & & & & + & \\
\hline Pentadecenol & & & & & & + & & \\
\hline x, y-Pentadecadien-1-ol & + & & & & & & & \\
\hline x-Pentadecen-1-ol & + & & & & & & & \\
\hline z, w-Pentadecadien-1-ol & + & & & & & & & \\
\hline Pentadecyl alcohol & & & & & & & & + \\
\hline 5-Tetradecen-1-ol & + & & & & & & & \\
\hline Tetradecanol & + & & & & & + & + & \\
\hline 4-Tridecen-1-ol & + & & & & & & & \\
\hline Tridecanol & + & & & & & + & + & \\
\hline Tridecenol & & & & & & & + & \\
\hline Tridecyl alcohol & & & & & & & & + \\
\hline Undecanol & & & & & & + & + & \\
\hline$\alpha$-Tocoferol & & + & & & & & & \\
\hline ÁCIDO CARBOXÍLICO & $\begin{array}{c}\text { A. } \\
\text { sinensis }\end{array}$ & $\begin{array}{c}A . \\
\text { mississippiensis } \\
\end{array}$ & $\begin{array}{c}\text { C. } \\
\text { crocodilus } \\
\end{array}$ & $\begin{array}{c}\text { C. } \\
\text { latirostris } \\
\end{array}$ & $\begin{array}{c}C . \\
\text { yacare }\end{array}$ & $\begin{array}{c}P . \\
\text { palpebrosus } \\
\end{array}$ & $\begin{array}{c}P . \\
\text { trigonatus } \\
\end{array}$ & $\begin{array}{c}\text { C. } \\
\text { acutus } \\
\end{array}$ \\
\hline z,9- Octanoic acid & + & & & & & & & \\
\hline Oleic acid & & & & & & & & + \\
\hline Heptadecanoic acid & + & & & & & & & \\
\hline Hexadecanoic acid & + & + & & & & + & + & \\
\hline Myristic acid & & & & & & & & + \\
\hline Octadecanoic acid & & + & & & & & & \\
\hline Palmitic acid & & & & & & & & + \\
\hline Pentadecanoic acid & + & & & & & + & & + \\
\hline Stearic acid & & & & & & & & + \\
\hline Tetradecanoic acid & + & & & & & & & \\
\hline Z-9- hexadecenoic acid & + & & & & & & & \\
\hline Z-9- octadecanoic acid & + & & & & & & & \\
\hline
\end{tabular}




\begin{tabular}{|c|c|c|c|c|c|c|c|c|}
\hline CETONA & $\begin{array}{c}\text { A. } \\
\text { sinensis }\end{array}$ & $\begin{array}{c}\text { A. } \\
\text { mississippiensis }\end{array}$ & $\begin{array}{c}\text { C. } \\
\text { crocodilus }\end{array}$ & $\begin{array}{c}\text { C. } \\
\text { latirostris }\end{array}$ & $\begin{array}{c}\text { C. } \\
\text { yacare }\end{array}$ & $\begin{array}{c}P . \\
\text { palpebrosus }\end{array}$ & $\begin{array}{c}\text { P. } \\
\text { trigonatus }\end{array}$ & $\begin{array}{c}\text { C. } \\
\text { acutus }\end{array}$ \\
\hline 3-Ethylheptadecan-2-one & & & + & & & & & \\
\hline 3-Ethylheptan-2-one & & & + & & + & & & \\
\hline 3-Ethylheptan-4-one & & & + & + & + & & & \\
\hline 3-Ethylnon-5-en-4-one & & & + & + & + & & & \\
\hline 3-Ethylnonan-2-one & & & + & + & + & & & \\
\hline 3-Ethylnonan-4-one & & & + & + & + & + & + & \\
\hline 3-Ethylnonane-2,4-dione & & & + & + & & & & \\
\hline 3-Ethylnonane-4,6-dione & & & + & & + & & + & \\
\hline 3-Ethylpentadecan-2-one & & & + & + & + & & + & \\
\hline 3-Ethyltridecan-2-one & & & + & & & & + & \\
\hline 3-Ethylundecan-2-one & & & & & & & + & \\
\hline 3-Etil-undecan-2-ona & & & + & & & & & \\
\hline 3-Etil-undecane-2,4-diona & & & + & & & & & \\
\hline 5-Ethyldec-2-en-4-one & & & + & + & & & & \\
\hline 5-Ethylhept-2-en-4-one & & & + & + & + & & & \\
\hline 5-Ethylheptane-2,4-dione & & & + & + & & & & \\
\hline 5-Ethylnon-2-em-4-one & & & + & + & + & + & + & \\
\hline 5-Ethylnonan-4-one & & & + & + & + & + & & \\
\hline 5-Ethylnonane-2,4dione & & & + & + & + & + & & \\
\hline 5-Ethyloct-2-em-4-one & & & & + & & + & & \\
\hline 5-Ethyloctan-4-one & & & + & + & & & & \\
\hline $\begin{array}{l}\text { 5-Ethylpendadec-2-en-4- } \\
\text { one }\end{array}$ & & & + & & + & & & \\
\hline \multicolumn{9}{|l|}{$\begin{array}{l}\text { 5-Ethylpentadecane-2,4- } \\
\text { dione }\end{array}$} \\
\hline 5-Ethyltridec-2-en-4-one & & & + & + & + & & & \\
\hline $\begin{array}{l}\text { 5-Ethyltridecane-2,4- } \\
\text { dione }\end{array}$ & & & + & & & & & \\
\hline 5-Ethylundec-2-en-4-one & & & + & + & + & + & & \\
\hline \multicolumn{9}{|l|}{ 5-Ethylundecan-4-one } \\
\hline 5-Ethylundecan-6-one & & & + & + & + & + & + & \\
\hline 5-Etil-undecane-2,4-diona & & & + & + & + & & & \\
\hline 7-Ethyldec-4-en-6-one & & & + & + & & & & \\
\hline 7-Ethyldodec-4-em-6-one & & & + & + & & & & \\
\hline $\begin{array}{l}\text { 7-Ethylheptadecane-4,6- } \\
\text { dione }\end{array}$ & & & + & & & & & \\
\hline 7-Ethylpentadec-4-en-one & & & + & & & & & \\
\hline 7-Ethyltridec-4-en-6-one & & & + & + & + & & & \\
\hline 7-Ethyltridecan-6-one & & & + & & & & & \\
\hline $\begin{array}{l}\text { 7-Ethyltridecane-4,6- } \\
\text { dione }\end{array}$ & & & + & + & + & & & \\
\hline 7-Ethylundec-4-en-6-one & & & + & + & + & + & + & \\
\hline $\begin{array}{l}\text { 7-Ethylundecane-4,6- } \\
\text { dione }\end{array}$ & & & + & + & + & & & \\
\hline $\begin{array}{l}\text { 7-Etil-heptadec-4-en-6- } \\
\text { ona }\end{array}$ & & & + & + & + & & & \\
\hline Heptan-3-one & & & & + & & & & \\
\hline ÁLDEIDOS & $\begin{array}{c}\text { A. } \\
\text { sinensis }\end{array}$ & $\begin{array}{c}\text { A. } \\
\text { mississippiensis }\end{array}$ & $\begin{array}{c}\text { C. } \\
\text { crocodilus }\end{array}$ & $\begin{array}{c}\text { C. } \\
\text { latirostris }\end{array}$ & $\begin{array}{c}C . \\
\text { yacare }\end{array}$ & $\begin{array}{c}P . \\
\text { palpebrosus }\end{array}$ & $\begin{array}{c}\text { P. } \\
\text { trigonatus }\end{array}$ & $\begin{array}{c}\text { C. } \\
\text { acutus }\end{array}$ \\
\hline 2-Ethyldecanal & & & + & + & & & & \\
\hline 2-Ethyldodecanal & & & + & + & + & & + & \\
\hline 2-Ethyloctanal & & & + & + & & & & \\
\hline 2-Ethyltetradecanal & & & + & + & + & + & + & \\
\hline HYDROCARBONETOS & $\begin{array}{c}\text { A. } \\
\text { sinensis }\end{array}$ & $\begin{array}{c}\text { A. } \\
\text { mississippiensis }\end{array}$ & $\begin{array}{c}\text { C. } \\
\text { crocodilus }\end{array}$ & $\begin{array}{c}\text { C. } \\
\text { latirostris }\end{array}$ & $\begin{array}{c}C . \\
\text { yacare }\end{array}$ & $\begin{array}{c}P . \\
\text { palpebrosus }\end{array}$ & $\begin{array}{c}P . \\
\text { trigonatus }\end{array}$ & $\begin{array}{c}\text { C. } \\
\text { acutus }\end{array}$ \\
\hline Cholesta-3,5-diene & & & & & & & & + \\
\hline Cholesterol & & + & & + & & & & + \\
\hline Sesquiterpeno & & & & + & & & & \\
\hline
\end{tabular}


The ethanolic extract of PG exudate of $C$. yacare had a MIC of $25 \mu \mathrm{g} / \mathrm{mL}$ and MFC of $50 \mu \mathrm{g} / \mathrm{mL}$ against $C$. albicans. In accordance with this result it is possible to affirm a antimicrobial function exerted by PG on $C$. yacare cloaca, which probably protects cloaca and neighboring structures from fungal infections. This statement makes sense since the natural history of $C$. yacare is closely associated with water and PG may be a natural passive defense of the organism.

ACKNOWLEDGMENTS: Financial support of FAPEMAT $\mathrm{n}$ 0 160048/2014.

\section{REFERENCES}

ALBERNAZ, L. C.; DE PAULA, J. E.; ROMERO, G. A.; SILVA, M. DO R.; GRELLIER, P.; MAMBU, L.; ESPINDOLA, L. S..

Investigation of plant extracts in traditional medicine of the Brazilian Cerrado against protozoans and yeasts. Journal of Ethnopharmacology, v. 131, n. 1, p. 116-121, 2010. DOI: http://doi.org/10.1016/j.jep.2010.06.011

ALVARENGA, A. L.; SCHWAN, R. F.; DIAS, D. R.; SCHWANESTRADA, K. R. F.; BRAVO-MARTINS, C. E. C.. Atividade antimicrobiana de extratos vegetais sobre bactérias patogênicas humanas. Rev. Bras. PI. Med., p.86-91, 2007.

ALVES, R. R. N.; VIEIRA, W. L. S.; SANTANA, G. G. Reptiles used in traditional folk medicine: conservation implications. Biodiversity and Conservation, v. 17, n. 8, p. 2037-2049, 1 jul. 2008. DOI: http://doi.org/10.1007/s10531-007-9305-0

ALVES, R. R. N.; ROSA, I. L. Trade of Animals Used in Brazilian Traditional Medicine: Trends and Implications for Conservation. Human Ecology, v. 38, n. 5, p. 691-704, 2010.

BARROS, B. F.; VARELA, A. M. S.; PEREIRA, M. H.; VICENTE, L... Medicinal use of fauna by a traditional community in the Brazilian Amazonia. Journal of Ethnobiology and Ethnomedicine, v. 8, n. 1, p. 1-19, 2012. DOI: http://doi.org/10.1186/1746-4269-8-37

BAYDAR, H.; SA, O.. Antibacterial activity and composition of essential oils from Origanum, Thymbra and Satureja species with commercial importance in Turkey. Food Control, v. 15, p. 169-172, 2004. DOI: http://doi.org/10.1016/S0956$\underline{\text { 7135(03)00028-8 }}$

BLAKE, D. K.; LOVERIDGE, J. P.. The role of commercial crocodile farming in crocodile conservation. Biological Conservation, v. 8, n. 4, p. 261-272, 1975. DOI: http://doi.org/10.1016/0006-3207(75)90004-X

BUTHELEZI, S.; SOUTHWAY, C.; GOVINDEN, U.; BODENSTEIN, J.; TOIT, D. K.. An investigation of the antimicrobial and antiinflammatory activities of crocodile oil. Journal of Ethnopharmacology, v. 143, n. 1, p. 325-330, DOI: http://doi.org/10.1016/j.jep.2012.06.040

CLSI. Reference method for broth dilution antifungal susceptibility testing of yeasts: approved standard - third edition. Clinical and Laboratory Standards Institute, n. April, p. $1-25,2008$

CLSI. Methods for Dilution Antimicrobial Susceptibility Tests for Bacteria That Grow Aerobically ; Approved Standard Ninth Edition. Methods for Dilution Antimicrobial
Susceptibility Tests for Bacteria That Grow Aerobically; Approved Standar- Ninth Edition. Anais..2012

DUNN, B. S. J.; WELDON, P. J.; HOWARD, R. W.; MCDANIEL, C. A.. A Lipids From the Paracloacal Glands of the Chinese Alligator (Alligator sinensis). American Oil Chemist's Society, v. 28 , n. 1, p. 75-78, 1993. DOI:

http://doi.org/10.1007/BF02536366

ELLOF, J. N.. A sensitive and quick Microplate Method to Determine the Minimum Inhibitory Sensisitive and Concentration of Plant Extracts for Bacteria. Planta Medica, v. 64, n. 2, p. 711-713, 1998. DOI: http://doi.org/10.1055/s2006-957563

FERNANDES, V. R. T.; SOUZA FRANCO, M. L.; MIKCHA, J. M; DE SOUZA, V. L.; GASPARINO, E.; COUTINHO, M. E.; TANAMATI, A.; DEL VESCO A. P.. Yacare caiman (Caiman yacare) trim hamburger and sausage subjected to different smoking techniques. Journal of the Science of Food and Agriculture, v. 94 , n. 3, p. 468-472, fev. 2014. DOI: http://doi.org/10.1002/jsfa.6270

GIAMARELLOU, H.. Treatment options for multidrugresistant bacteria. Future drugs, v. 4, n. 4, p. 601-18, 2006. DOI: http://doi.org/10.1586/14787210.4.4.601

GARCÍA-RUBIO, S.; ATTYGALLE, A. B.; WELDON, P. J.; MEINWALD, J.. Reptilian chemistry: volatile compounds from paracloacal glands of the American crocodile (Crocodylus acutus). Journal of chemical ecology, v. 28, n. 4, p. 769-781, 2002. DOI: http://doi.org/10.1023/A:1015288726605

GUIMARÃES, D. O.; MOMESSO, L. S.; PUPPO, M. T.. Antibióticos: Importância terapêutica e perspectivas para a descoberta e desenvolvimento de novos agentes. Química Nova, v. 33, n. 3, p. 667-679, 2010.

HOLETZ, F. B.; PESSINI, G. L.; SANCHES, N. R.; CORTEZ, D. A. G.; NAKAMURA, C. V.; FILHO, B. P. D.. Screening of some plants used in the Brazilian folk medicine for the treatment of infectious diseases. Memórias do Instituto Oswaldo Cruz, v. 97, n. 7, p. 1027-1031, out. 2002. DOI: http://doi.org/10.1590/S0074-02762002000700017

INOUYE, S.; UCHIDA, K.; MARUYAMA, N.; YAMAGUCHI, H.; $A B E$, S. et al.. A Novel Method to Estimate the Contribution of the Vapor Activity of Essential Oils in Agar Diffusion Assay. Japanese Journal of Medical Mycology, v. 47, 2006.

JUNIOR, N. L. S.. A regulamentação de acesso a recursos genéticos e repartição de benefícios: Disputas dentro e fora 
do Brasil. Ambiente \& Sociedade, v. 14, n. 1, p. 229-244, 2010. DOI: http://doi.org/10.1590/S1414$\underline{753 \times 2011000100013}$

KATE, K. T and LAIRD, S. A., 1999.. The commercial use of biodiversity: Access to gentic resources and benefit-sharing. 1a ed. UK: Earthscan. 168p.

KOMMANEE, J.; PREECHARRAM, S.; DADUANG, S.; TEMSIRIPONG, Y.; DHIRAVISIT, A.; YAMADA, Y.; THAMMASIRIRAK, S.. Antibacterial activity of plasma from crocodile (Crocodylus siamensis) against pathogenic bacteria. Annals of clinical microbiology and antimicrobials, v. 11 , n. 1, p. 22, jan. 2012. Dol http://doi.org/10.1186/1476-0711-11-22

KOMMANEE, J.; Phosri, S.; Daduang, S.; Temsiripong, Y.; Dhiravisit, A.; Thammasirirak, S.. Comparisons of antiinflammatory activity of crocodile (Crocodylus siamensis) blood extract. Chiang Mai Journal of Science, v. 41, n. 3, p. 627-634, 2014

KRUCKERT, K.; FLACHSBARTH, B.; SCHULZ, S.; HENTSCHEL, U.; WELDON, P. J.. Ehtyl-branched Adehydes, ketones, and Diketones from Caimans (Caiman and Paleosuchus; Crocodylia, Reptilia). Natural Products, v. 69, n. 6, 2006. DOI: http://doi.org/10.1021/np0600797

LIMA, I. DE O.; OLIVEIRA, R. DE A. G.; LIMA, E, DE O.; FARIAS, N. M. P.; SOUZA, E. L.. Atividade antifúngica de óleos essenciais sobre espécies de Candida. Brazilian Journal of Pharmacognosy, v. 16, n. 2, p. 197-201, 2006.

MCDANIEL, C. J.; Dunn, B. J.; Weldon, P. J.. Citronellol and Citronellyl Esters from the Paracloacal Glands of the BroadSnouted Caiman, Caiman latirostris ( Crocodylia, Reptilia). Z. Naturforsch, p. 932-934, 1998. DOI:

http://doi.org/10.1515/znc-1998-9-1026

MENALE, B.; DE CASTRO, O.; CASCONE, C.; MUOIO, R.. Ethnobotanical investigation on medicinal plants in the Vesuvio National Park (Campania, Southern Italy). Journal of Ethnopharmacology, v. 192, p. 320-349, 2016. DOI: http://doi.org/10.1016/j.jep.2016.07.049

MERCHANT, M.; THIBODEAUX, D.; LOUBSER, K.; ELSEY, R. M. Amoebacidal effects of serum from the American alligator (Alligator mississippiensis). Journal of Parasitology, v. 90, n. 6, p. 1480-1483, 2004. DOI: http://doi.org/10.1645/GE-3382

MERCHANT, M. E.; ROCHE, C.; ELSEY, R. M.; PRUDHOMME, J.. Antibacterial properties of serum from the American alligator (Alligator mississippiensis). Comparative Biochemistry and Physiology Part B: Biochemistry and Molecular Biology, v. 136, n. 3, p. 505-513, nov. 2003. DOI: http://doi.org/10.1016/S1096-4959(03)00256-2

MERCHANT, M. E.; LEGER, N.; JERKINS, E.; MILLS, K.; PALLANSCH, M. B.; PAULMAN, R. L.; PTAK, R. G.. Broad spectrum antimicrobial activity of leukocyte extracts from the American alligator (Alligator mississippiensis). Veterinary immunology and immunopathology, v. 110, n. 3-4, p. 2218, 15 abr. 2006. DOI: http://doi.org/10.1463/1948020

MORAIS, C. S. N.; MORAIS, J. N. N.; VICENTE-NETO, J.; RAMOS, E. Z.; ALMEIDA, J.; ROSEIRO, C.; SANTOS, C.; GAMA, L. T.; BRESSAN, M. C.. Mortadella sausage manufactured with Caiman yacare (Caiman crocodilus yacare) meat, pork backfat, and soybean oil. Meat Science, v. 95, n. 2, p. 403411, out. 2013. DOI:

http://doi.org/10.1016/j.meatsci.2013.04.017

MOURÃO, G.. DE M. Utilização econômica da fauna silvestre no Brasil: O exemplo do jacaré-do-pantanal. EMBRAPA, v. 5, p. $1-4,2000$

NASCIMENTO, P. F. C.; NASCIMENTO, A. C.; RODRIGUES, C. S.. Atividade antimicrobiana dos óleos essenciais : uma abordagem multifatorial dos métodos. Brazilian Journal of Pharmacognosy, v. 17, n. 1, p. 108-113, 2007. DOI: http://doi.org/10.1590/S0102-695X2007000100020

NATECHE, F. et al. Application of the resazurin microtitre assay for detection of multidrug resistance in Mycobacterium tuberculosis in Algiers. Journal of Medical Microbiology, v. 55, n. 7, p. 857-860, 2006. DOI: http://doi.org/10.1099/jmm.0.46513-0

OLIVEIRA, E. S.; TORRES, D. F.; BROOKS, S. E.; ALVES, R. R.N.. The medicinal animal markets in the metropolitan region of Natal City, northeastern Brazil. Journal of Ethnopharmacology, v. 130, n. 1, p. 54-60, 2010. DOI: http://doi.org/10.1016/j.jep.2010.04.010

OLIVEIRA, M. M. M..; BRUGNERA, D. F.; CARDOSO, M. G.; GUIMARÃES, L. G. L.; PICCOLI, R. H.. Rendimento , composição química e atividade antilisterial de óleos essenciais de espécies de Cymbopogon. Rev. Bras. PI. Med., v. 13, n. 1, p. 8-16, 2011. DOI:

http://doi.org/10.1128/AAC.76.8.2763267-224422.678002

PALOMINO, J. C. MARTIN, A.; CAMACHO, M.; GUERRA, H.; SWINGS, J.; PORTAELS, F.. Resazurin Microtiter Assay Plate : Simple and Inexpensive Method for Detection of Drug Resistance in Mycobacterium tuberculosis. Antimicrobial Agents and Chemotherapy, v. 46, n. 8, p. 2720-2722, 2002. DOI: http://doi.org/10.1128/AAC.46.8.2720-2722.2002

PATA, S.; YARAKSA, N.; DADUANG, S.; TEMSIRIPONG, Y.; SVASTI, J.; ARAKI, T.; THAMMASIRIRAK, S.. Characterization of the novel antibacterial peptide Leucrocin from crocodile (Crocodylus siamensis) white blood cell extracts.

Developmental and comparative immunology, v. 35, n. 5, p. 545-53, maio 2011. DOI: http://doi.org/10.1016/j.dci.2010.12.011

PINTO, A. C.; SILVA, D. H. S.; BOLZANI, V. DA S.; LOPES, N. P.; EPIFANIO, R. DE A.. Produtos naturais: Atualidade, desafios e perspectivas. Química Nova, v. 25, n. SUPPL. 1, p. 45-61, 2002. DOI: http://doi.org/10.1590/S010040422002000800009

ROMANELLI, P. F.; CASERI, R.; FILHO, J. F.. Processamento da carne do jacaré (Caiman crocodilus yacare). Ciência e Tecnologia de Alimentos, v. 22, n. 1, p. 70-75, 2002. DOI: http://doi.org/10.1590/S0101-20612002000100013

SANT'ANA, P. J. P.. As parcerias para a bioprospecção no Brasil. Parcerias Estratégicas, v. 15, p. 111-127, 2002.

SANTOS, A. P.; ZATTA, D. T.; MORAES, W. F.; BARA, M. T. F.; SILVA, M. DO R. R.; PAULA, J. R.. Composição química , atividade antimicrobiana do óleo essencial e ocorrência de esteróides nas folhas de Pterodon emarginatus Vogel, 
Fabaceae. Brazilian Journal of Pharmacognosy, 2010. DOI: http://doi.org/10.1590/S0102-695X2010005000052

SOUTO, W. M. S. et al. Parallels between zootherapeutic practices in ethnoveterinary and human complementary medicine in northeastern Brazil. Journal of

Ethnopharmacology, v. 134, n. 3, p. 753-767, 2011. DOI: http://doi.org/10.1016/j.jep.2011.01.041

TORTORA, G. J.; CASE, C. L.; FUNKE, B. R. Microbiologia. 10. ed. Artmed: Porto Alegre, 2012. VERDADE, L. M.. A exploração da fauna silvestre no Brasil: jacarés, sistemas e recursos humanos. Biota Neotrópica, v. 4, n. 2, p. 1-12, 2004. DOI: http://doi.org/10.1590/S167606032004000200002

WELDON, P. J.; SCOTT, T. P.; TANNER, M. J.. Analysis of Gular and Paracloacal Gland secretions of the American Alligator (Alligar mississippiensis) by Thin-Layer Chromatography. Journal of chemical ecology, v. 16, n. 1, p. 3-12, 1989. DOI: http://doi.org/10.1007/BF01021263

WELDON, P. J.; SHAFAGATI, A.; WHEELER, J. W.. Lipids from the paracloacal glands of the American alligator (Alligator mississippiensis). Lipids, v. 23, n. 7, p. 727-729, jul. 1988. DOI: http://doi.org/10.1007/BF02535676
WELDON, P. J.; SHAFAGATI, A.; WHEELER, J. W.. Lipids on the paracloacal gland secretions of Dwarf (Paleosuchus palpebrosus) and Smooth-fronted (P.trigonatus) Caimans. Biochemical Systematics and Ecology, v. 17, n. 5, p. 431435, 1989. Dol: http://doi.org/10.1016/03051978(89)90061-6

WILLIAMS, P. A Mitchell, W.; Wilson, G. R.; Weldon, P.. J.Bacteria in the Gular and paracloacal glands of the American alligator (Alligator mississippiensis; Reptilia, Crocodila). Letters in Applied Microbiology, v. 10, p. 73-79, 1989. DOI: http://doi.org/10.1111/j.1472765X.1990.tb00268.x

WHEELER, J. W.; IBRAHIM, A.; P. WELDON, J.. 2,3Dihydrofarnesyl and citronellyl esters in the paracloacal gland secretions of the brown caiman (Caiman crocodilus fuscus) from Costa Rica. Biochemical Systematics and Ecology, v. 27, n. 1, p. 27-32, jan. 1999. DOI: http://doi.org/10.1016/S0305-1978(98)00054-4

ZHANG, Z.; ELSOHLY, H. N.; JACOB, M. R.; PASCO, D. S.; WALKER, L. A.; CLARK, A. M.. Natural products inhibiting Candida albicans secreted aspartic proteases from Lycopodium cernuum. Journal of Natural Products, v.65, n.7, p.979-985, 2002. DOI: http://doi.org/10.1007/BF025374956

A CBPC - Companhia Brasileira de Produção Científica (CNPJ: 11.221.422/0001-03) detém os direitos materiais desta publicação. Os direitos referem-se à publicação do trabalho em qualquer parte do mundo, incluindo os direitos às renovações, expansões e disseminações da contribuição, bem como outros direitos subsidiários. Todos os trabalhos publicados eletronicamente poderão posteriormente ser publicados em coletâneas impressas sob coordenação da Sustenere Publishing, da Companhia Brasileira de Produção Científica e seus parceiros autorizados. Os (as) autores (as) preservam os direitos autorais, mas não têm permissão para a publicação da contribuição em outro meio, impresso ou digital, em português ou em tradução. 